SUPPORTING INFORMATION

\title{
Resolving a Fluorescence Quenching Mechanism of an Oxazine Dye Using Ultrabroadband Two-Dimensional Electronic Spectroscopy
}

William P. Carbery, Brismar Pinto-Pacheco, Daniela Buccella, * and Daniel Turner*

Department of Chemistry, New York University, New York, New York 10003

Section

Page Number

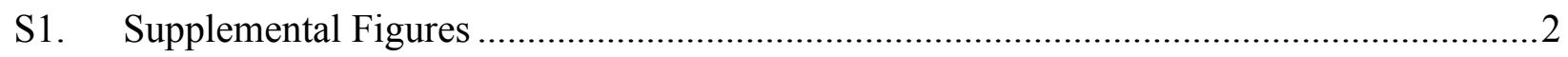

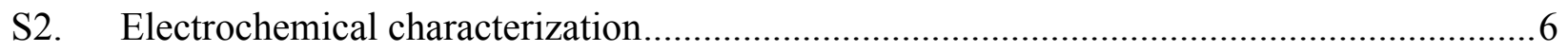

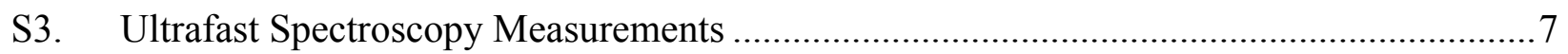

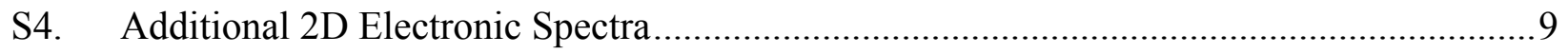

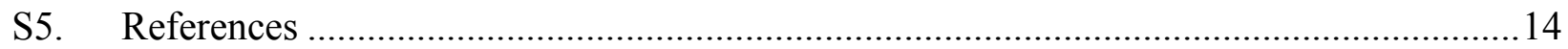




\section{S1. Supplemental Figures}
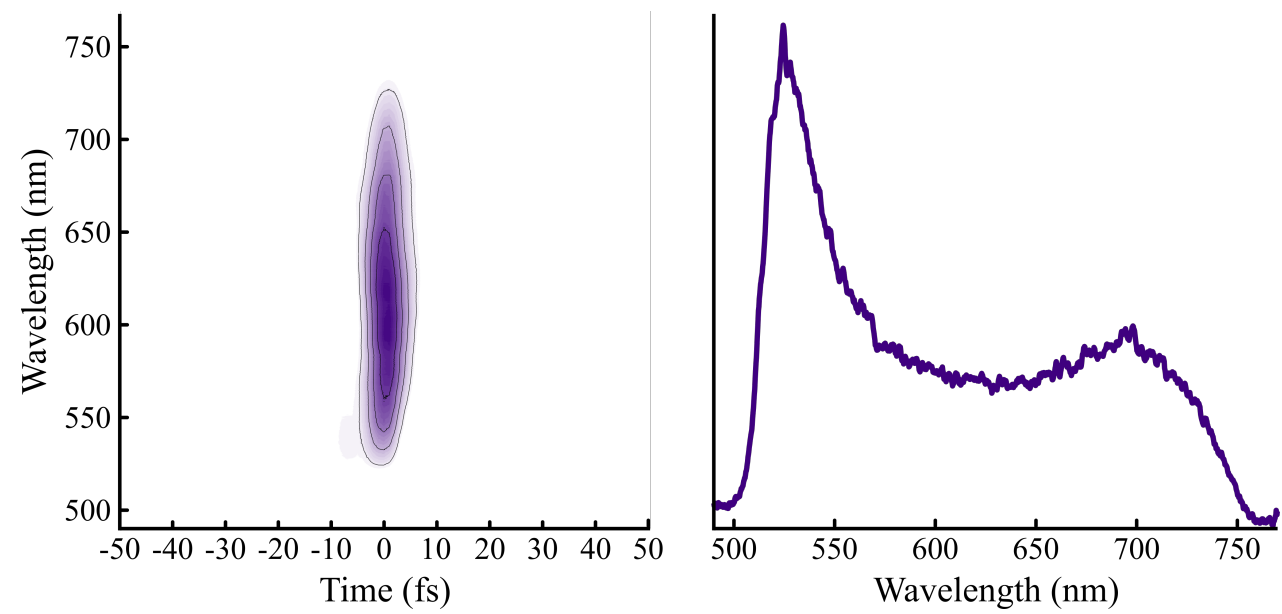

Figure S1. TG-FROG (left) and NOPA spectrum (right) characterizing the pulse used for the 2D ES measurements reported here. The NOPA pulse is compressed using a prism-based pulse shaper to $6.6 \mathrm{fs}$ in duration.

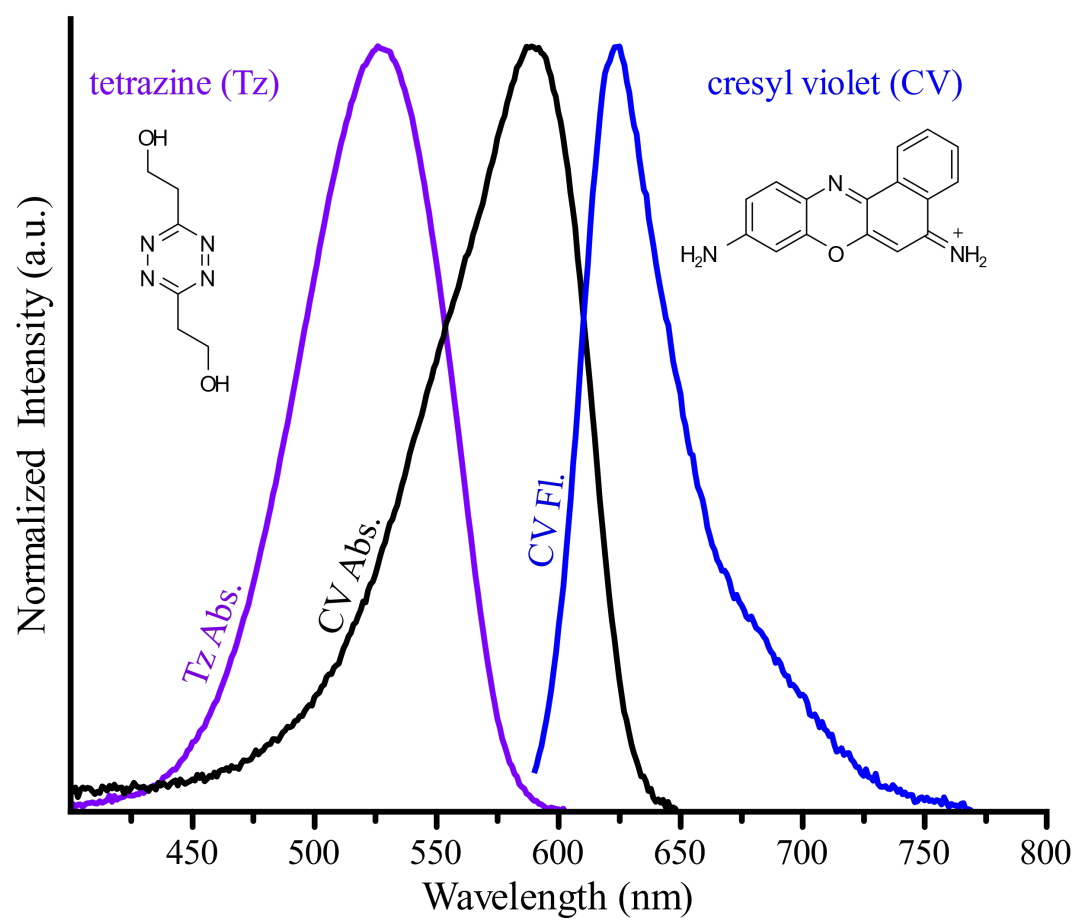

Figure S2. Linear absorption (black) and fluorescence (blue) spectra of cresyl violet perchlorate $(\mathrm{CV}+)$ along with the linear absorption (purple) of tetrazine $(\mathrm{Tz})$ in 2:1 acetonitrile/PBS buffer. 


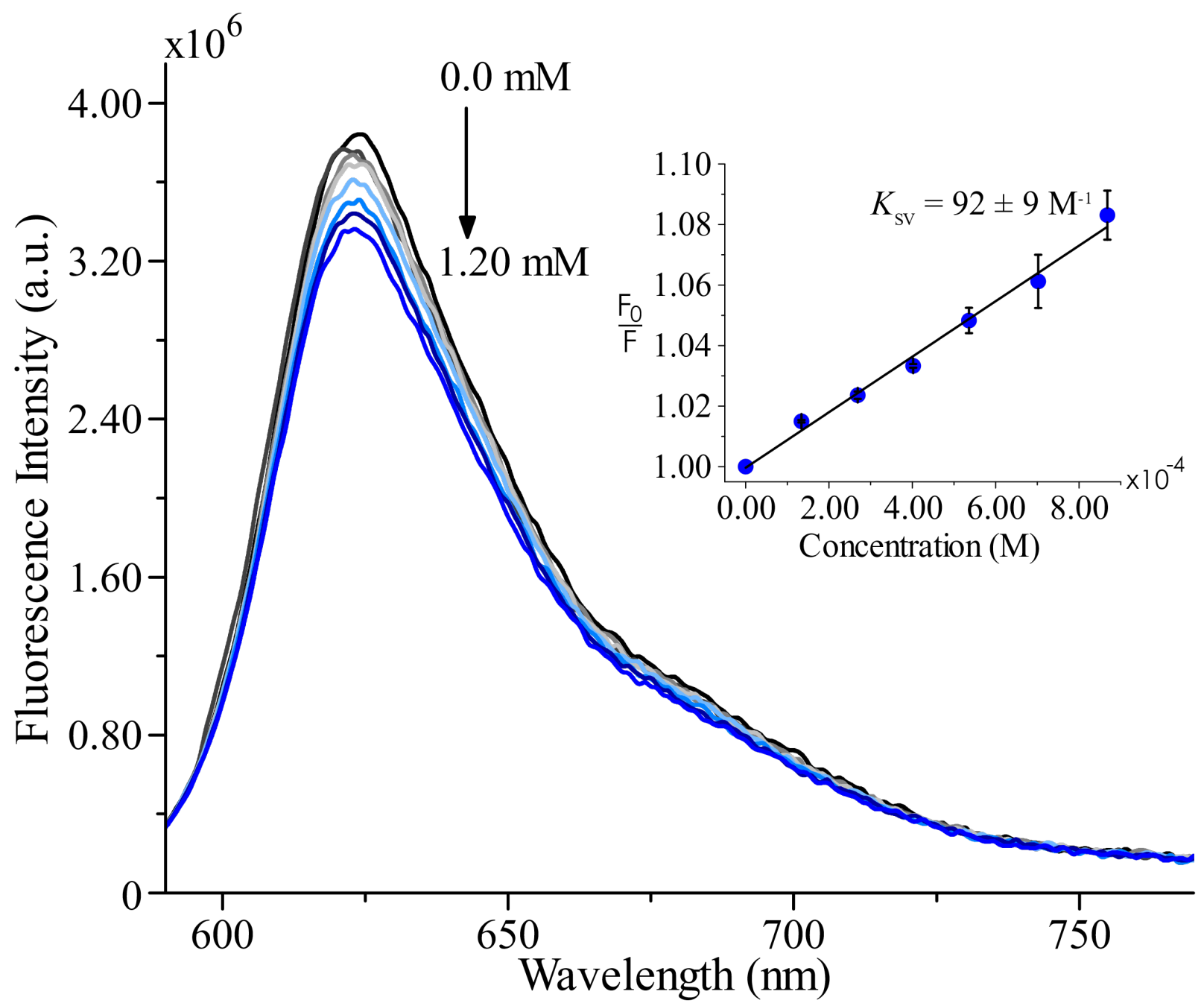

Figure S3. Fluorescence spectra of cresyl violet with increasing concentrations of tetrazine and resulting steady state Stern-Volmer plot (inset). The $K_{S V}$ value obtained for this system was $92 \pm$ $9 \mathrm{M}^{-1}$. 


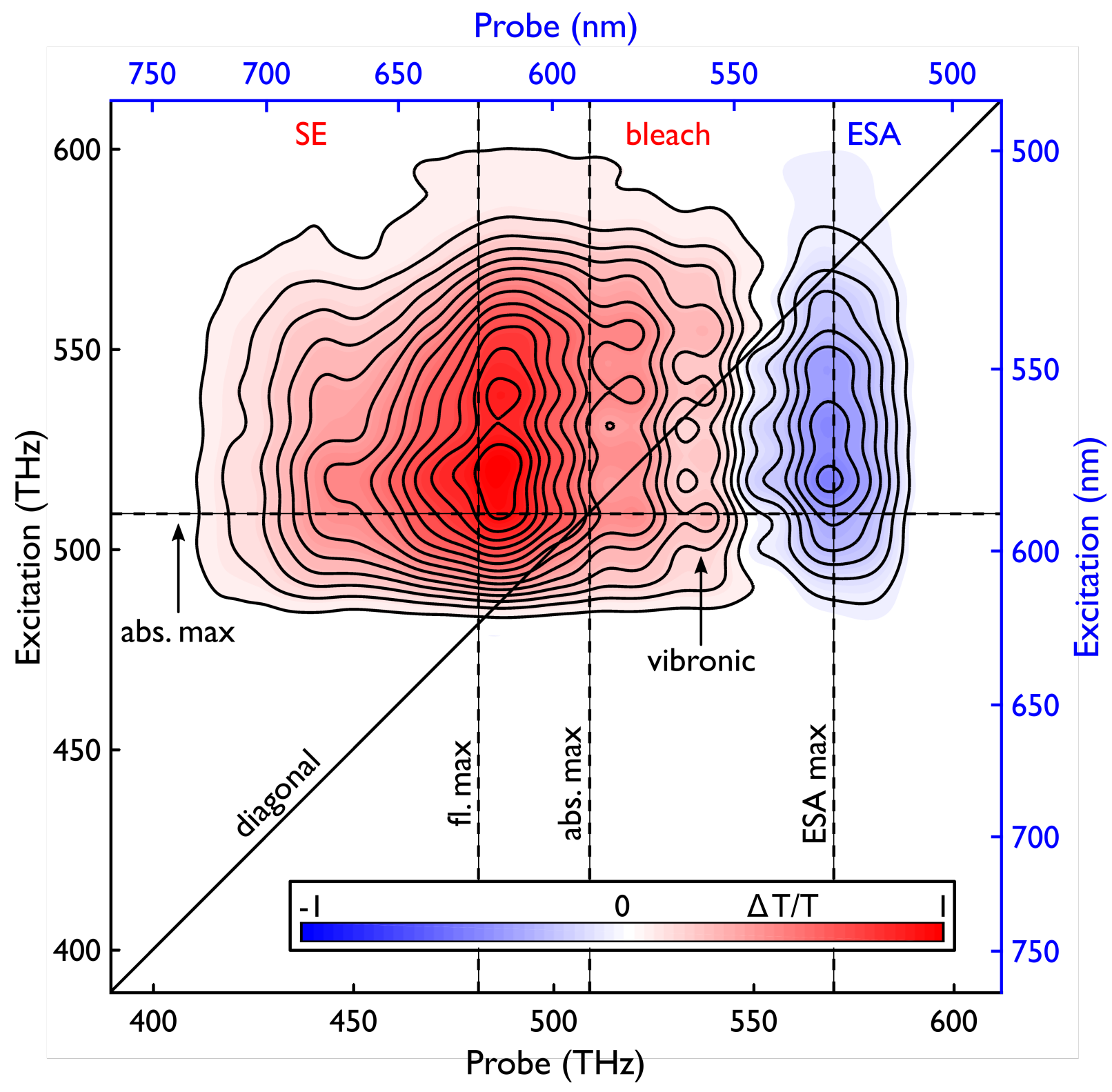

Figure S4. Sample 2D electronic spectra of cresyl violet at a waiting time of $100 \mathrm{ps,}$, with axes in $\mathrm{THz}$ and nm units. All 2D electronic spectra are normalized relative to the highest intensity bleach/SE peak such that the change in transmittance $(\Delta \mathrm{T} / \mathrm{T})$ is reported from -1 (ESA) to 1 (bleach/SE). 


\section{Cresyl Violet}
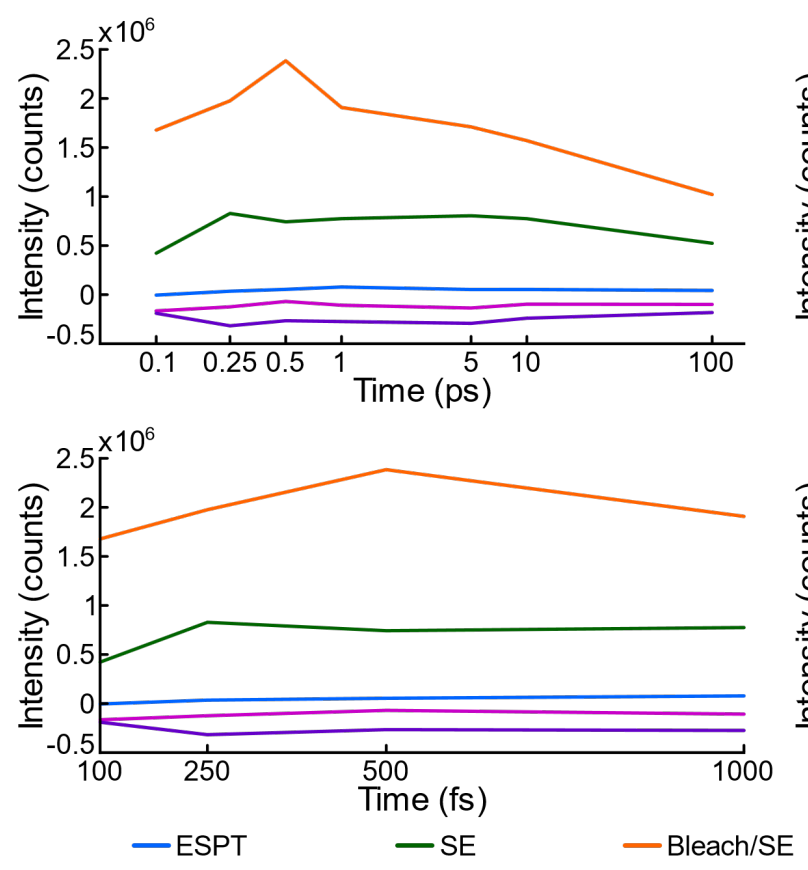

Cresyl Violet and Tetrazine
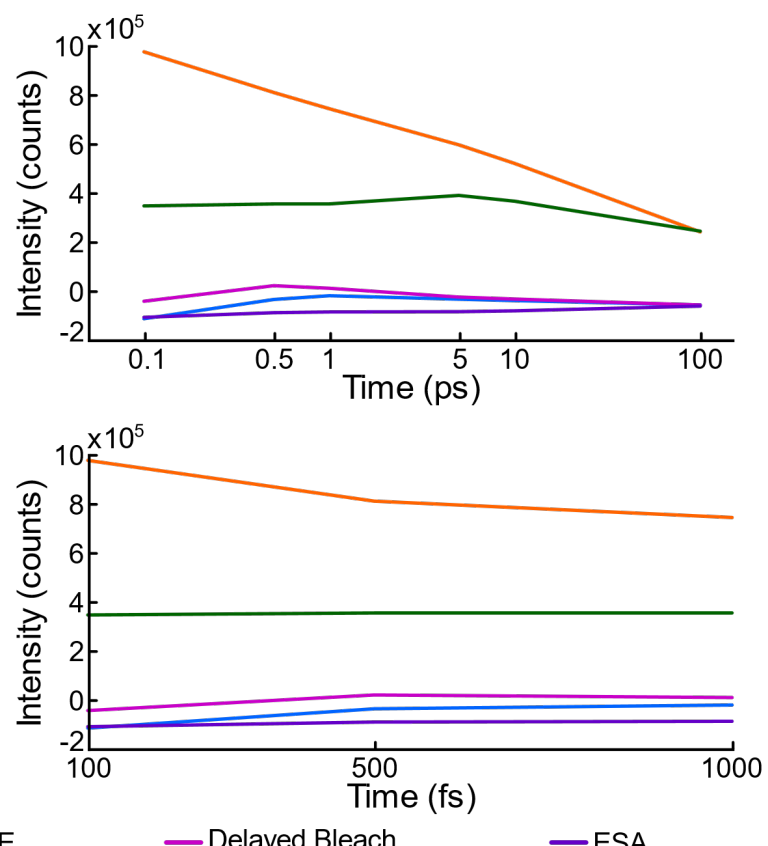

-Delayed Bleach -ESA

Figure S5. Kinetic plots obtained from summing the ESPT (blue), SE (green), bleach and SE (orange), delayed bleach (magenta), and ESA (purple) regions at specific waiting times. The picosecond plots (top) include a logarithmic time scale, while the zoomed-in plots (bottom) are linear. Generally the change in intensity for the first $100 \mathrm{ps}$ is small for the ESA and delayed bleach plots, although both show that same initial rise and the same timescale as the proposed ESPT region. The kinetics of the main bleach and SE peak have strong vibrational modulations at early times, but are generally consistent with a short rise time (for the SE contribution) and long decay time ( $>200 \mathrm{ps}$ ) that corresponds to the 3.2 nanosecond fluorescence lifetime of cresyl violet. 


\section{S2. Electrochemical characterization}

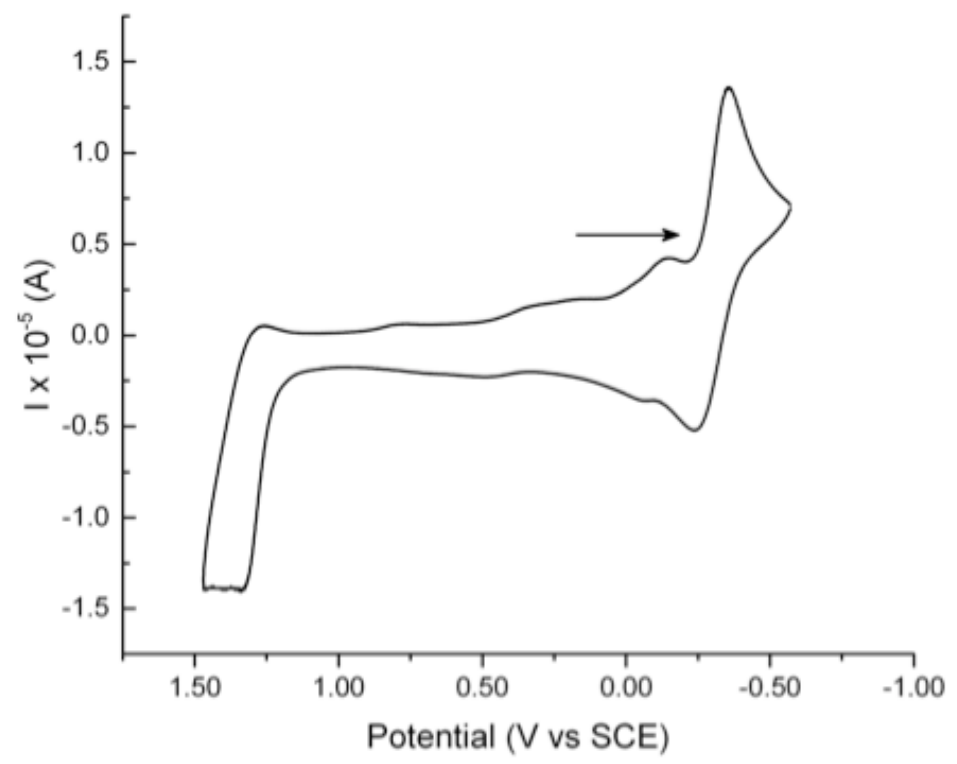

Figure S5. Cyclic voltammogram of $1 \mathrm{mM}$ cresyl violet perchlorate in $0.1 \mathrm{M}$ TBAF in acetonitrile. A reversible behavior was observed for the reduction with a $57 \mathrm{mV} / \mathrm{s}$ difference between the anodic and the cathodic peak. In the case of the oxidation, an irreversible peak was observed.

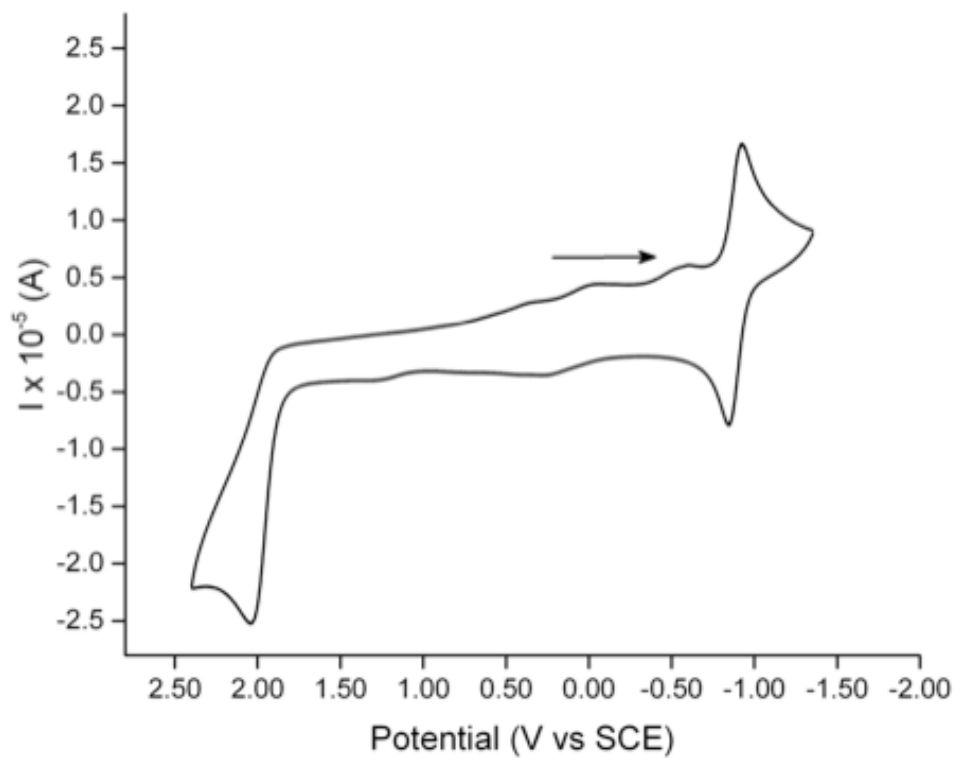

Figure S6. Cyclic voltammogram of $1 \mathrm{mM}$ 3,6-di(2-hydroxyethyl)-1,2,4,5-tetrazine in $0.1 \mathrm{M}$ TBAF in acetonitrile. Two single-step electron transfer reductions were observed. The first reduction, reversible, at $-0.874 \mathrm{~V}$ vs. SCE with a peak-to-peak separation of $78 \mathrm{mV} / \mathrm{s}$ and the second reduction, irreversible, at $2.045 \mathrm{~V}$ vs. SCE. The results are in agreement with redox behavior of other 1,2,4,5-tetrazines reported in literature. ${ }^{1-2}$ 


\section{S3. Ultrafast Spectroscopy Measurements}

Briefly, a Ti:sapphire oscillator (80 MHz, 790 nm, $30 \mathrm{~nm}$ FWHM, 8nJ) seeds a Ti:sapphire amplifier to yield laser pulses at a $1.25 \mathrm{kHz}$ repetition rate $(11 \mathrm{~nm}, \mathrm{FWHM}, 700 \mu \mathrm{J}, \sim 0.1 \%$ shotto-shot RSD). The output pumps a custom built noncolinear optical parametric amplifier (NOPA) that results in broadband laser pulses of 500-760 nm at $200 \mathrm{~nJ}$ and with $\sim 0.5$ shot-toshot RSD. These are subsequently compressed using a prism-based pulsed shaper to 6.6 fs as measured by transient-grating frequency resolved optical gating (TG-FROG). For transient absorption (TA) and two-dimensional electronic spectroscopy (2D ES) measurements, the compressed laser pulse is split into four beams in the BOX geometry. The probe/LO pulse is attenuated with a neutral density filter to be $10^{4}$ less intense than the three pump beams. ${ }^{3-4}$

For initial measurements of cresyl violet and tetrazine a UV-vis spectrum taken before and after the experiment (approximately $18 \mathrm{hrs}$ of irradiation) showed no signs of degradation. However, long-term irradiation slowly degrades the tetrazine such that the fluorescence quenching effect is no longer observed. 


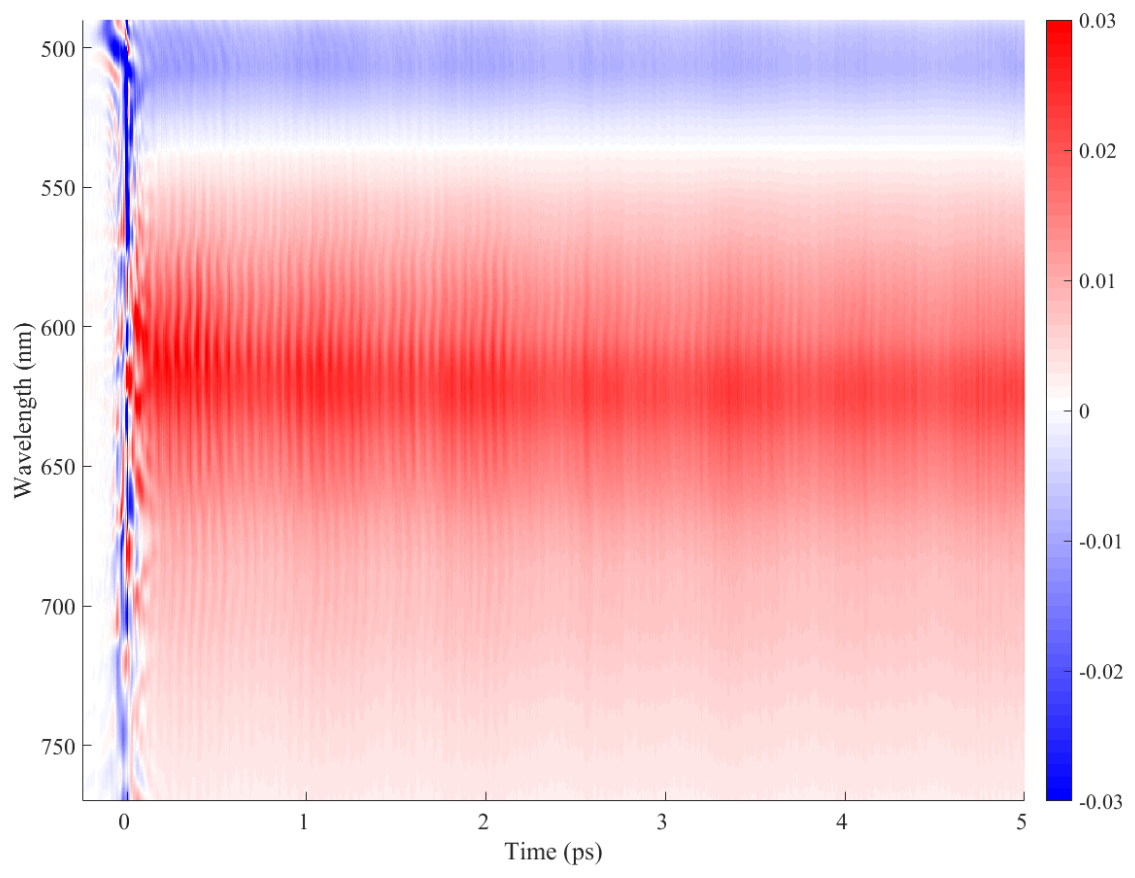

Figure S7. Transient absorption spectra of cresyl violet perchlorate in $2: 1$ acetonitrile/PBS buffer from 0 to $5 \mathrm{ps}$.

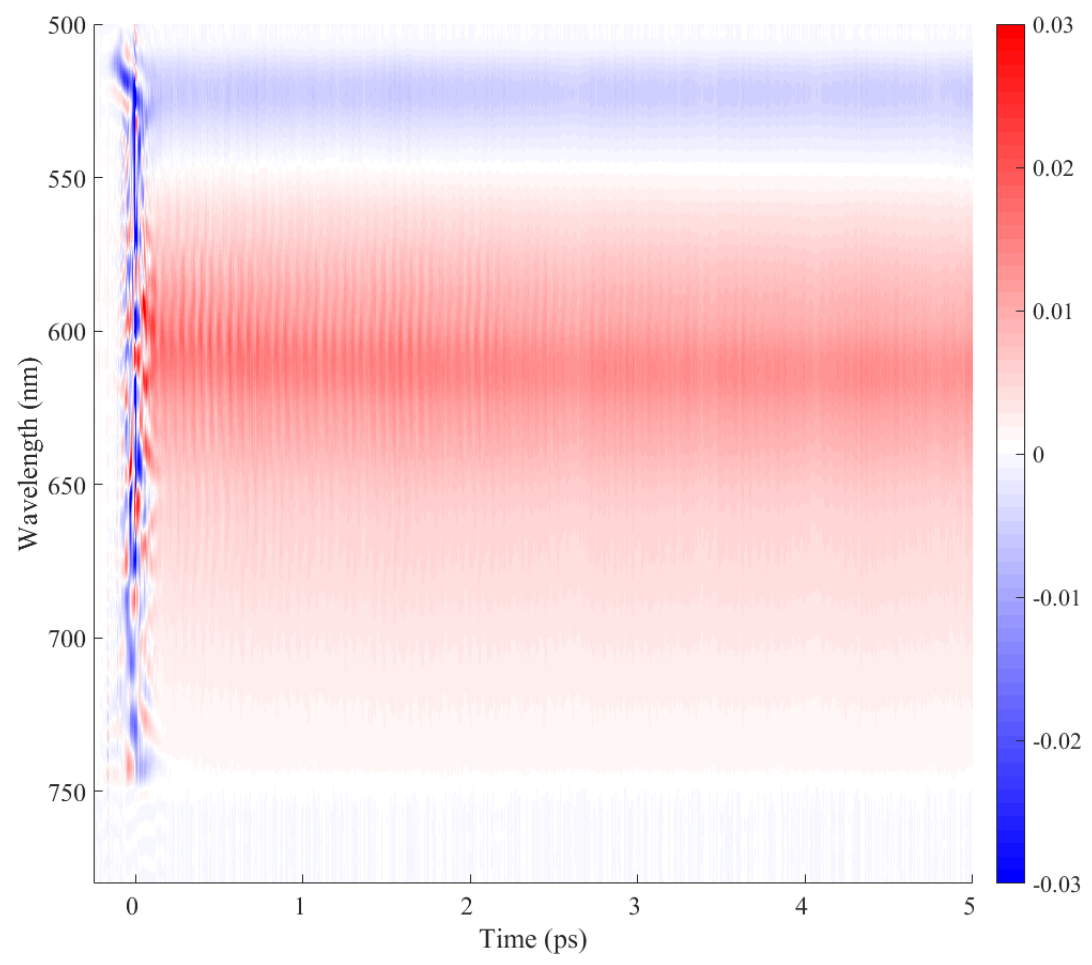

Figure S8. Transient absorption spectra of cresyl violet perchlorate mixed with tetrazine in 2:1 acetonitrile/PBS buffer from 0 to 5 ps. 


\section{S4. Additional 2D Electronic Spectra}
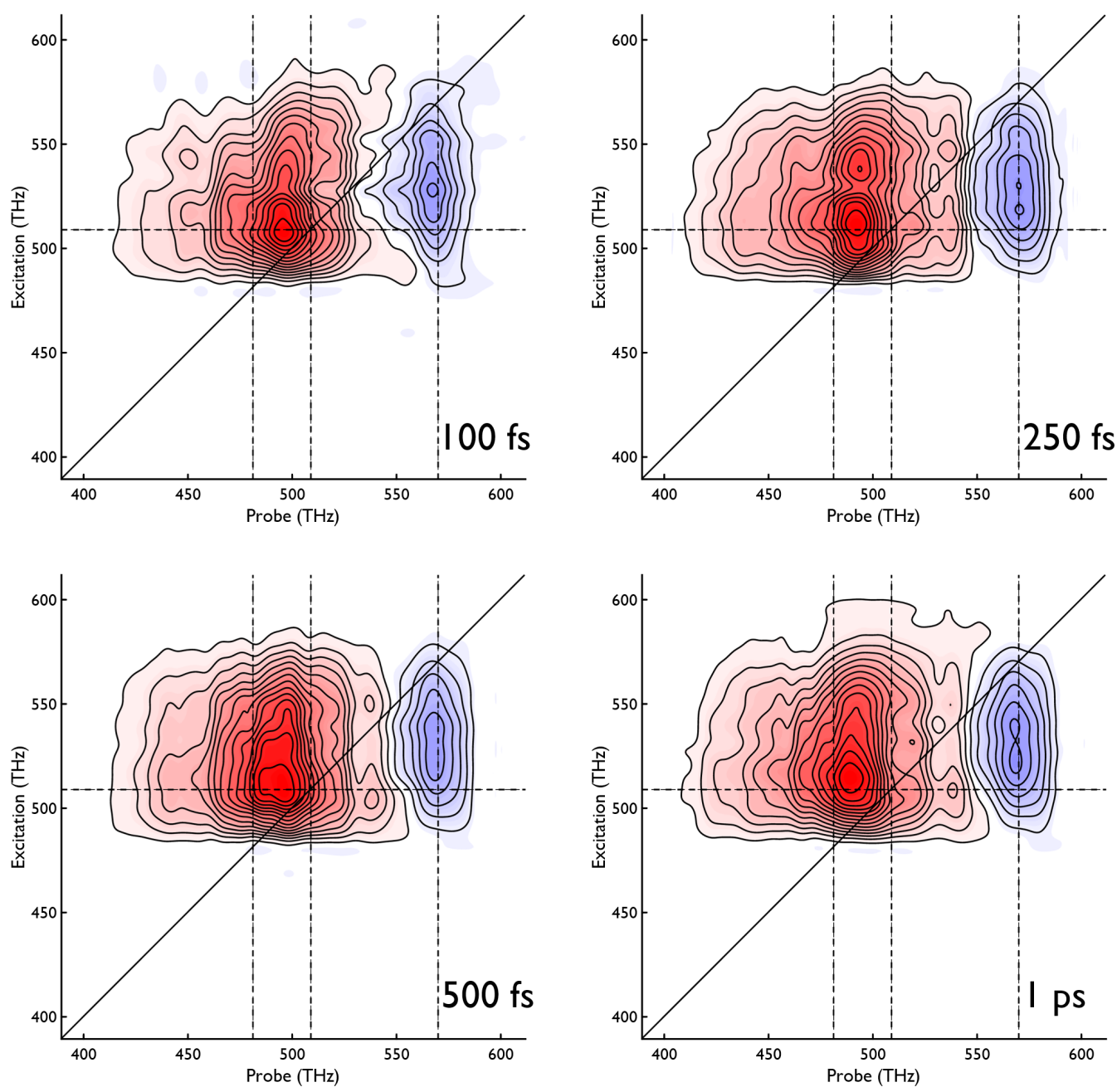

Figure S9. Additional 2D electronic spectra of cresyl violet perchlorate in 2:1 acetonitrile/PBS buffer at waiting times of $100 \mathrm{fs}, 250 \mathrm{fs}, 500 \mathrm{fs}$, and $1 \mathrm{ps}$. The color scale matches the cresyl violet map (Fig. S3), where each spectrum is normalized to the highest intensity peak. 

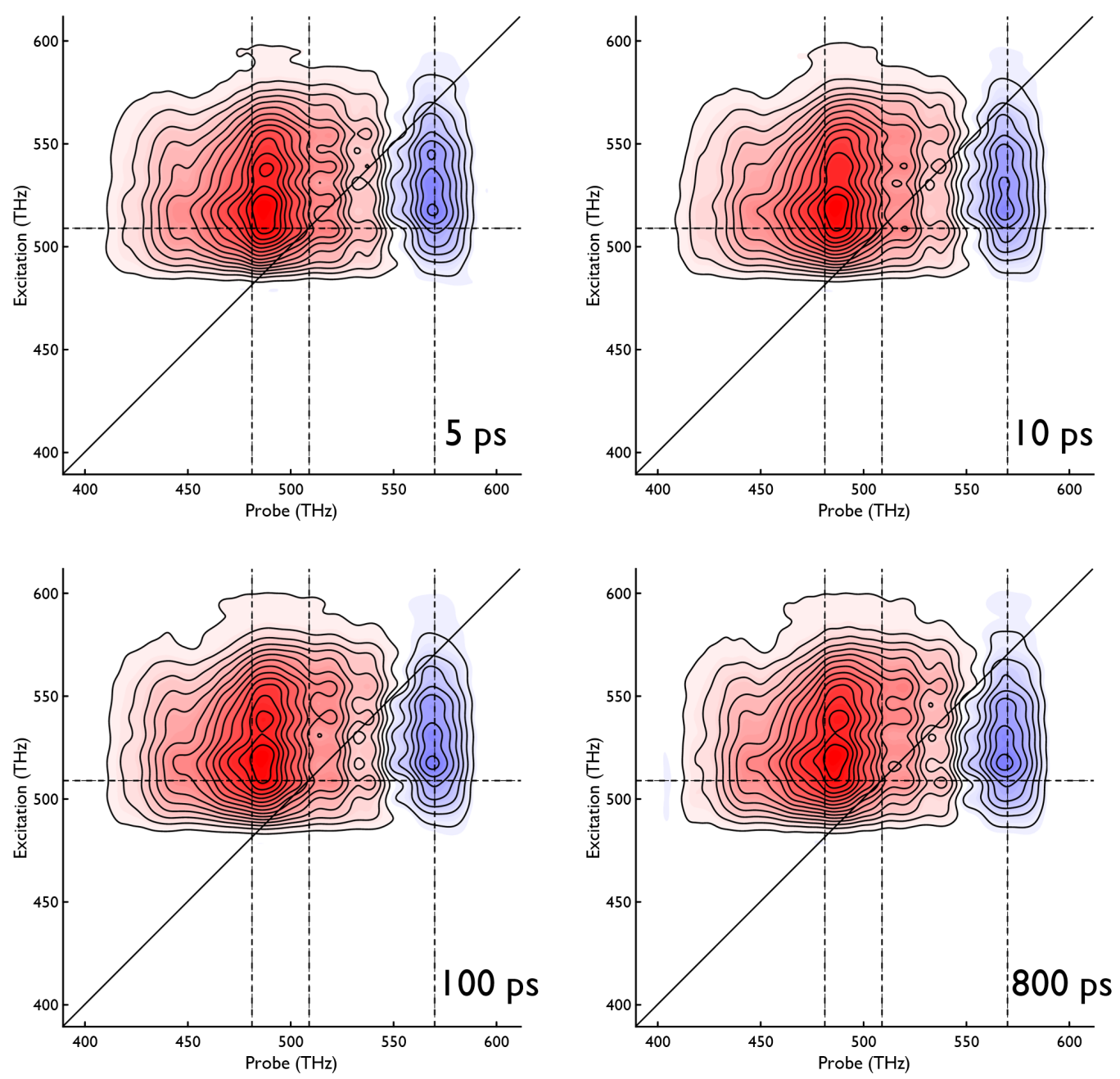

Figure S10. Additional 2D electronic spectra of cresyl violet perchlorate in 2:1 acetonitrile/PBS buffer at waiting times of $5 \mathrm{ps}, 10 \mathrm{ps}, 100 \mathrm{ps}$, and $800 \mathrm{ps}$. The color scale matches the cresyl violet map (Fig. S3), where each spectrum is normalized to the highest intensity peak. 

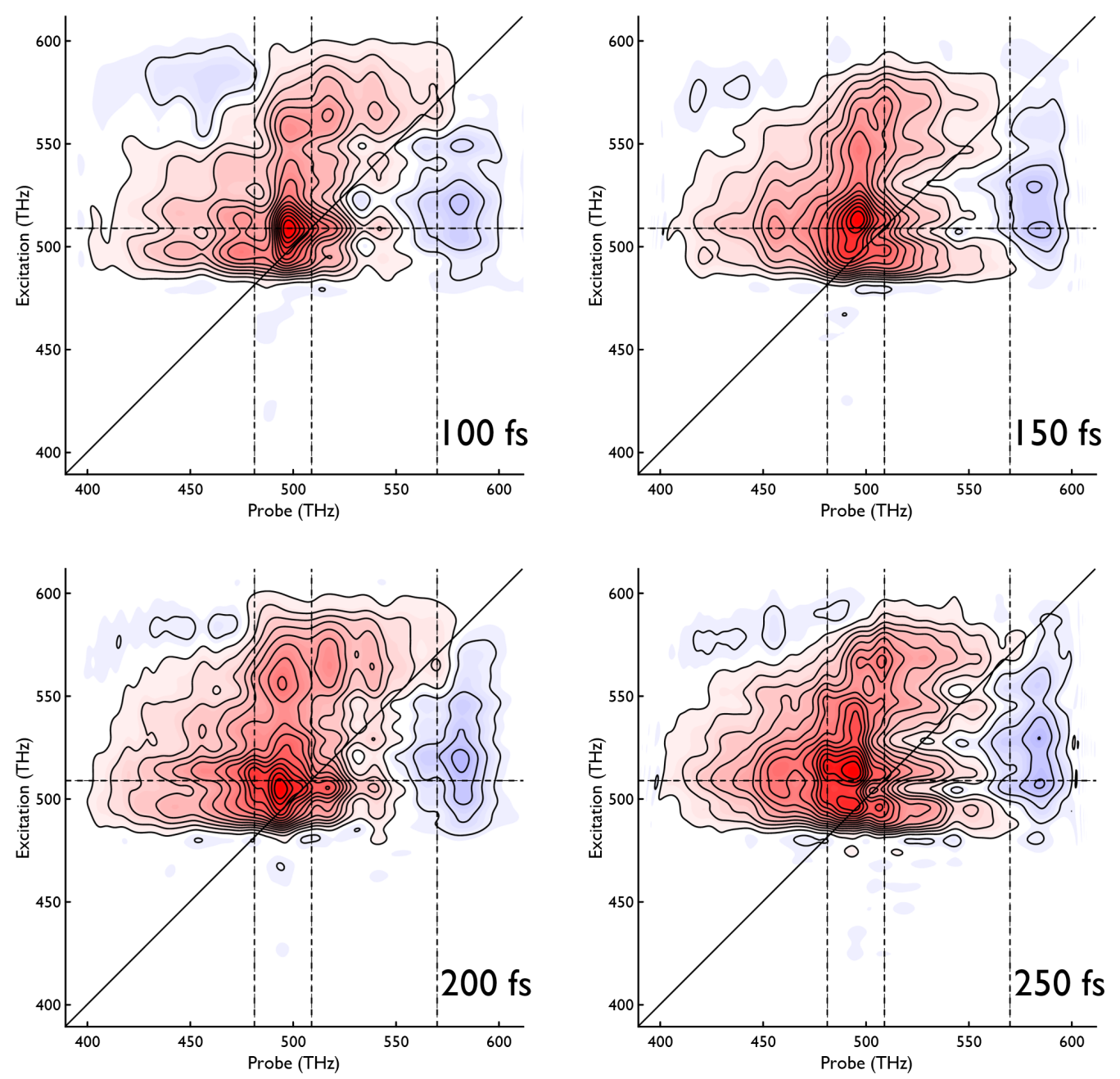

Figure S11. Additional 2D electronic spectra of cresyl violet perchlorate mixed with tetrazine in 2:1 acetonitrile/PBS buffer at waiting times of $100 \mathrm{fs}, 150 \mathrm{fs}, 200 \mathrm{fs}$, and $250 \mathrm{fs}$. The color scale matches the cresyl violet map (Fig. S3), where each spectrum is normalized to the highest intensity peak. 

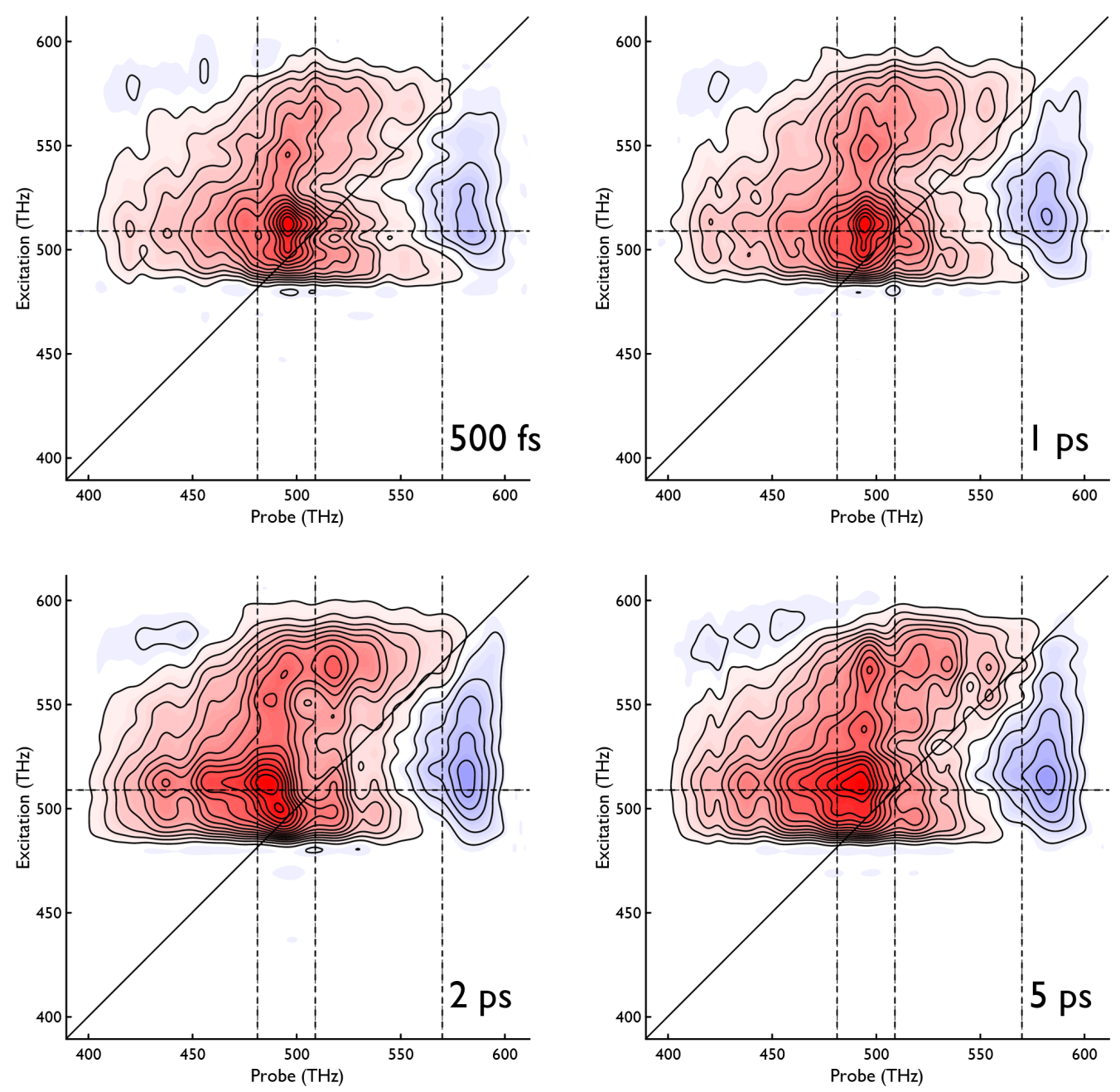

Figure S12. Additional 2D electronic spectra of cresyl violet perchlorate mixed with tetrazine in 2:1 acetonitrile/PBS buffer at waiting times of $500 \mathrm{fs}, 1 \mathrm{ps}, 2 \mathrm{ps}$, and $5 \mathrm{ps}$. The color scale matches the cresyl violet map (Fig. S3), where each spectrum is normalized to the highest intensity peak. 

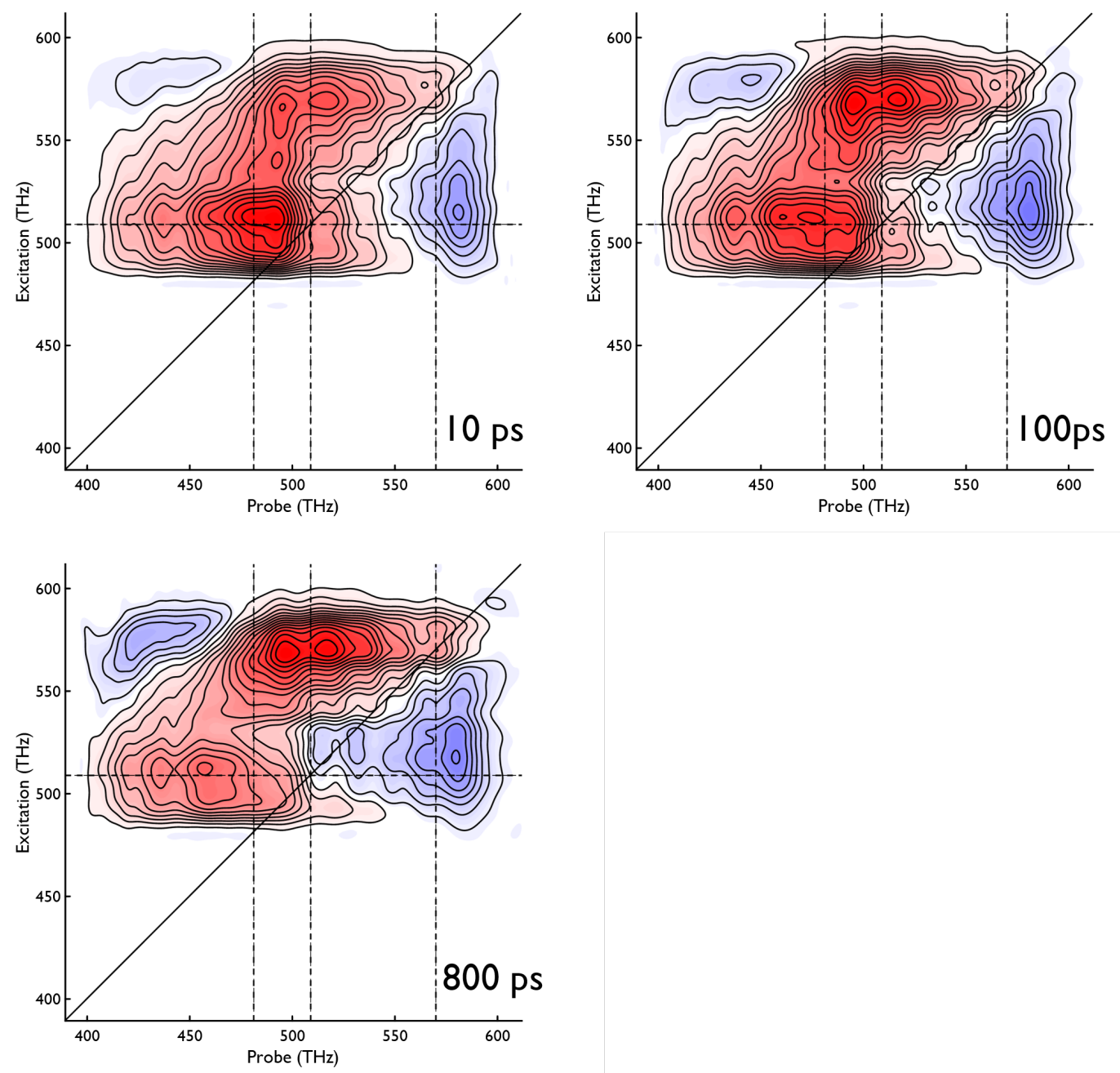

Figure S13. Additional 2D electronic spectra of cresyl violet perchlorate mixed with tetrazine in 2:1 acetonitrile/PBS buffer at waiting times of $10 \mathrm{ps}, 100 \mathrm{ps}$, and $800 \mathrm{ps}$. The color scale matches the cresyl violet map (Fig. S3), where each spectrum is normalized to the highest intensity peak. 


\section{S5. References}

1. Ehret, F.; Wu, H.; Alexander, S. C.; Devaraj, N. K., Electrochemical Control of Rapid Bioorthogonal Tetrazine Ligations for Selective Functionalization of Microelectrodes. J. Am. Chem. Soc. 2015, 137 (28), 8876-8879.

2. Kaim, W., The coordination chemistry of 1,2,4,5-tetrazines. Coord. Chem. Rev. 2002, $230(1-2), 127-139$.

3. Brazard, J. Bizimana, L. A.; Turner, D. T., Accurate Convergence of TransientAbsorption Spectra Using Pulsed Lasers. Rev. Sci. Instrumen. 2015, 86 (5), 053106.

4. Bizimana, L. A.; Brazard, J.; Carbery, W. P.; Gellen, T.; Turner, D. B., Resolving Molecular Vibronic Structure Using High-Sensitivity Two-Dimensional Electronic Spectroscopy. J. Chem. Phys. 2015, 143 (16), 164203. 